\title{
ANÁLISES COMPARATIVAS ENTRE INDICADORES BIBLIOMÉTRICOS COM BASE NA PRODUÇÃO ACADÊMICA DE DOCENTES DO CURSO DE PÓS- GRADUAÇÃO DE ENGENHARIA DE PRODUÇÃO DA UNIVERSIDADE FEDERAL FLUMINENSE
}

\author{
Fernanda Luz da Silva
}

Pós Graduação em Engenharia de Produção, Universidade Federal Fluminense Rua Passo da Pátria 156, São Domingos, 24210-240, Niterói, RJ fluz@idd.uff.br

\section{Luana Carneiro Brandão}

Pós Graduação em Engenharia de Produção, Universidade Federal Fluminense Rua Passo da Pátria 156, São Domingos, 24210-240, Niterói, RJ luanabrandao@id.uff.br

\section{João Carlos Correia Baptista Soares de Mello}

Departamento de Engenharia de Produção - Universidade Federal Fluminense Rua Passo da Pátria 156, São Domingos, 24210-240, Niterói, RJ luanabrandao@id.uff.br

\section{RESUMO}

Por sua importância e simplicidade, o índice h, indicador bibliométrico proposto em 2005, é bastante popular na literatura, havendo centenas de estudos publicados com críticas e propostas de novos índices, os quais são conhecidos como índices tipo h. O presente artigo estuda alguns desses índices propostos para amenizar a insensibilidade do índice $\mathrm{h}$ a publicações muito citadas, a saber, os índices g, A e R. Primeiramente, estuda-se o comportamento de tais índices, frente a um aumento do nível de produção acadêmica, bem como a sua estabilidade, diante de variações na distribuição de citações. Para isso, utiliza-se como estudo de caso as publicações e citações dos docentes do curso de pós-graduação da Universidade Federal Fluminense.

Palavra-chave: Índice h; Índices Bibliométricos; Comparações entre índices.

\begin{abstract}
The h-index, proposed in 2005, has become very popular in the literature, due to its importance and simplicity. In fact, many studies propose new indexes, known as h-type indexes. This work studies a few of these indexes, which decrease the h-index's disadvantage of being insensitive to highly cited publications, namely the $\mathrm{g}$, A and $\mathrm{R}$ indexes. First, we compare the the indexes' reactions to an increase in the level of academic production, as well as their stability, when the citation distribution varies. For that, we considered the publications and citations of professors in the post graduation programme at Fluminense Federal University
\end{abstract}

Keywords: h-index; Bibliometric indexes; Comparisons between indexes. 


\section{Como Citar:}

SILVA, Fernanda Luz da; BRANDÃO, Luana Carneiro; MELLO, João Carlos Correia Baptista Soares de. Análises comparativas entre indicadores bibliométricos com base na produção acadêmica de docentes do curso de pós-graduação de Engenharia de Produção da Universidade Federal Fluminense. In: SIMPÓSIO DE PESQUISA OPERACIONAL E LOGÍSTICA DA MARINHA, 19., 2019, Rio de Janeiro, RJ. Anais [...]. Rio de Janeiro: Centro de Análises de Sistemas Navais, 2019.

\section{INTRODUÇÃO}

O índice bibliométrico proposto por Hirsch (2005) para medir impacto e relevância da produção científica, denominado índice h, é um dos indicadores mais populares na área da ciência da informação, provavelmente por sua importância, simplicidade e fácil compreensão (Franceschini e Maisano, 2010). O índice h é, não somente, muito estudado pela literatura, mas também, bastante utilizado informalmente, em diversas áreas associadas à pesquisa científica (Egghe, 2010).

Dentre as centenas de estudos relativos ao índice h, muitos apresentam críticas, e.g. Bornmann e Daniel (2007), Braun et al. (2006), Harzing e Van der Wal (2008), Norris e Oppenheim (2010), Panaretos e Malesios (2009), e propõem índices alternativos para superar as desvantagens, e.g., Alonso et al. (2010), Anderson et al. (2008), Batista et al. (2006), Cabrerizo et al. (2010), Egghe e Rousseau (2008), Schreiber (2008), Tol (2009).

O presente artigo realiza análises comparativas com o índice h e com alguns desses índices alternativos, chamados índices tipo h, quais sejam, os índices g (Egghe, 2006), A (Jin, 2006) e R (Jin et al., 2007). Esses índices buscam reduzir a insensibilidade do índice h a publicações muito citadas e não consideram outras variáveis além daquelas do índice h, quais sejam, o número de publicações e de citações de cada pesquisador. A principal contribuição deste trabalho é ampliar o conhecimento das características dos índices tipo h, para auxiliar instituições de ensino na escolha dos indicadores mais adequados em cada situação.

As análises comparativas do presente estudo são divididas em duas categorias, quais sejam, o comportamento dos índices frente a um aumento da produção do pesquisador e sua estabilidade. Na primeira categoria, são analisados os efeitos de um aumento do número de citações, somente, utilizando-se um exemplo hipotético. Em seguida, considera-se um estudo de caso real, qual seja, a produção dos docentes do curso de Pós-Graduação da Universidade Federal Fluminense (UFF), em dois períodos distintos, no intuito de avaliar os índices frente a um aumento de citações e publicações. Na segunda categoria, analisa-se a estabilidade dos índices, utilizando-se também o referido estudo de caso.

Na próxima seção, é feita uma revisão dos índices bibliométricos estudados. Na seção seguinte, são comparadas as reações dos índices frente a um aumento da produção de pesquisadores, com base em um exemplo ilustrativo e com base em um estudo de caso real. $\mathrm{Na}$ quarta seção, analisa-se a estabilidade dos índices, utilizando-se também o referido estudo de caso. Por fim, a quinta seção apresenta as conclusões.

\section{REVISÃO DE ALGUNS ÍNDICES BIBLIOMÉTRICOS}

\section{1. ÍNDICE $\mathbf{H}$}

Proposto por Hirsch (2005) para suprir a necessidade de avaliar o desempenho de pesquisadores, o índice h mede, de maneira simples e intuitiva, o impacto e relevância da 
produção científica. Por definição, um pesquisador apresenta o índice no valor de h, se o mesmo é autor de h publicações com, pelo menos, h citações cada, enquanto as demais publicações apresentam no máximo h citações. As h publicações com pelo menos h citações compõem o chamado núcleo do índice h.

Muitos trabalhos apontaram desvantagens desse índice, como o fato de não considerar a idade das publicações, o que beneficia pesquisadores antigos (BiHui, 2007; BiHui et al. 2007; Sidiropoulos et al., 2007; Franceschini e Maisano, 2010) e ser insensível a publicações muito citadas (Egghe, 2006, 2010; Schreiber, 2010b).

Algumas críticas levaram a propostas de novos índices tipo h que consideram outras variáveis, além do número de publicações e citações, como a idade da publicação, o tempo de carreira do pesquisador, etc. Assim, os índices que consideram apenas as variáveis originalmente propostas por Hirsch (2005), são ditos clássicos (Egghe, 2010). No presente estudo, são analisados apenas índices clássicos e que buscam superar a crítica de insensibilidade às publicações muito citadas.

\section{2. Í́NDICE G}

Uma das variantes mais populares do índice h (Franceschini e Maisano, 2010), o índice g é definido como o maior número natural de publicações que juntas receberam ao menos g $^{2}$ citações (Egghe, 2006), as quais formam o chamado núcleo g. Em outras palavras, o índice equivale ao número de publicações citadas, em média, pelo menos g vezes (Schreiber, 2010a). Dessa forma, o índice g valoriza publicações bastante citadas, sendo sempre maior ou igual do que o índice h, por definição (Egghe, 2010). Brandão \& Soares de Mello (2015) demonstram em que situações tais índices se equivalem.

Dentre as críticas do índice g, destaca-se a questão da precisão de dados (Jin et al, 2007). Isso porque o mesmo apresenta usualmente núcleos maiores do que o índice h, dependendo de um conjunto maior de informações precisas para que seja adequadamente calculado. Assim, foi proposto o índice A para lidar com a insensibilidade do índice h, sem acrescentar problemas de precisão.

\section{3. Í́NDICE $\mathbf{A}$}

O índice A é definido como o número médio de citações das publicações do núcleo h (Jin, 2006), como em (1), onde h é o número de publicações no núcleo h e $\mathrm{c}_{\mathrm{j}}$ é o número de citações para cada publicação $\mathrm{j}=1, \ldots, \mathrm{h}$. Observa-se que $\mathrm{A} \geq \mathrm{h}$ (Jin et al, 2007), uma vez que h é o número mínimo de citações do núcleo (podendo ser, ainda, menor que esse número mínimo), enquanto A é a média dessas citações.

$$
A=\frac{\sum_{j=1}^{p_{h}} c_{j}}{h} A=\frac{\sum_{j=1}^{h} c_{j}}{h}
$$

Tal índice apresenta certa similaridade com o índice g, uma vez que ambos são o valor médio de citações dentre um conjunto de publicações, embora o índice g seja um número natural e o $\mathrm{A}$, um número real. Além disso, os conjuntos de publicações são diferentes, sendo o núcleo $\mathrm{h}$ para o índice $\mathrm{A}$, e o núcleo do próprio índice g para este. Ressalte-se que $A \geq g$, conforme demonstrado em Jin et al. (2007). Essa relação pode ser 
observada pelo fato de o núcleo de g ser sempre maior ou igual que o núcleo de h, podendo englobar, assim, publicações menos citadas. Assim, a média de citações das publicações no núcleo h será sempre maior ou igual à média de citações das publicações no núcleo g.

Entretanto, o índice A não aumenta monotonicamente com o aumento das citações. Em outras palavras, é possível que um pesquisador receba novas citações e tenha o seu índice reduzido. Isso porque as novas citações podem levar a um aumento do índice h e, com isso, o seu novo núcleo pode apresentar uma média de citações menor. Essa característica indesejável é consequência da divisão por h (Jin et al., 2007), como observado em (1). Para lidar com essa questão, foi proposto o índice R.

\section{4. ÍNDICE $\mathbf{R}$}

O índice R é definido como a raiz do número total de citações das publicações do núcleo h (Jin et al, 2007), como em (2), em que as variáveis são as mesmas de (1).

$$
R=\sqrt{\sum_{j=1}^{h} c_{j}}
$$

Como não se trata de uma divisão por h, mas sim de uma função que depende somente da soma de citações no núcleo $h$, o índice $\mathrm{R}$ cresce monotonicamente com o aumento do número de citações. Assim, R não apresenta as desvantagens de $\mathrm{A}$, mas apresenta sensibilidade a publicações muito citadas, e considera o mesmo núcleo $h$ de publicações.

Observa-se, ainda, que $R \geq h$, uma vez que $c_{j} \geq h$ (Jin et al, 2007). Além disso, $A \geq R$. Para isso, basta rescrever (1) como $A \cdot h=\sum_{j=1}^{h} c_{j}$ e (2) como $R^{2} i \sum_{j=1}^{h} c_{j} R \cdot R=\sum_{j=1}^{h} c_{j}$, o que leva a $A . h=R^{2}$. Como $h \leq R$, então, para se manter a equação, tem-se $A \geq R$.

Em compensação, não é possível definir uma relação entre g e R. Geralmente, temse $\mathrm{g} \geq \mathrm{R}$. Isso porque os índices podem ser rescritos da seguinte forma: $R^{2} i \sum_{j=1}^{h} c_{j} R \cdot R=\sum_{j=1}^{h} c_{j}$ e $g^{2} \cong \sum_{j=1}^{g} c_{j}$. Neste último caso, o sinal não é de igualdade, por se tratar de um número natural, e não real. Como $\sum_{j=1}^{h} c_{j} \leq \sum_{j=1}^{g} c_{j}$, dado que $\mathrm{g} \geq \mathrm{h}$, tem-se, via de regra, $\mathrm{g} \geq \mathrm{R}$. Na verdade, se os índices apresentassem a mesma natureza numérica, seria garantido que $\mathrm{g} \geq \mathrm{R}$. Entretanto, se os valores das somas de citações nos núcleos g e h forem próximos, é possível ter $\mathrm{g}<\mathrm{R}$.

\section{AUMENTO DA PRODUÇÃO ACADÊMICA}

Nessa seção, analisa-se como os índices se comportam quando há um aumento do nível de produção dos pesquisadores. Avalia-se, primeiramente, o efeito do crescimento apenas de citações de forma hipotética e, posteriormente, um estudo de caso real, em que houve aumento, tanto do número de citações, quanto de publicações.

\subsection{CRescimento de CitAÇões - SituaÇão Hipotética}

Nesta primeira seção, avalia-se o comportamento dos índices estudados no presente 
artigo, frente a um crescimento do número de citações para as publicações do núcleo h. Ressalte-se que essa situação ocorre na prática quando um pesquisador cessa as suas atividades, mas continua tendo as suas publicações citadas, em especial as mais populares.
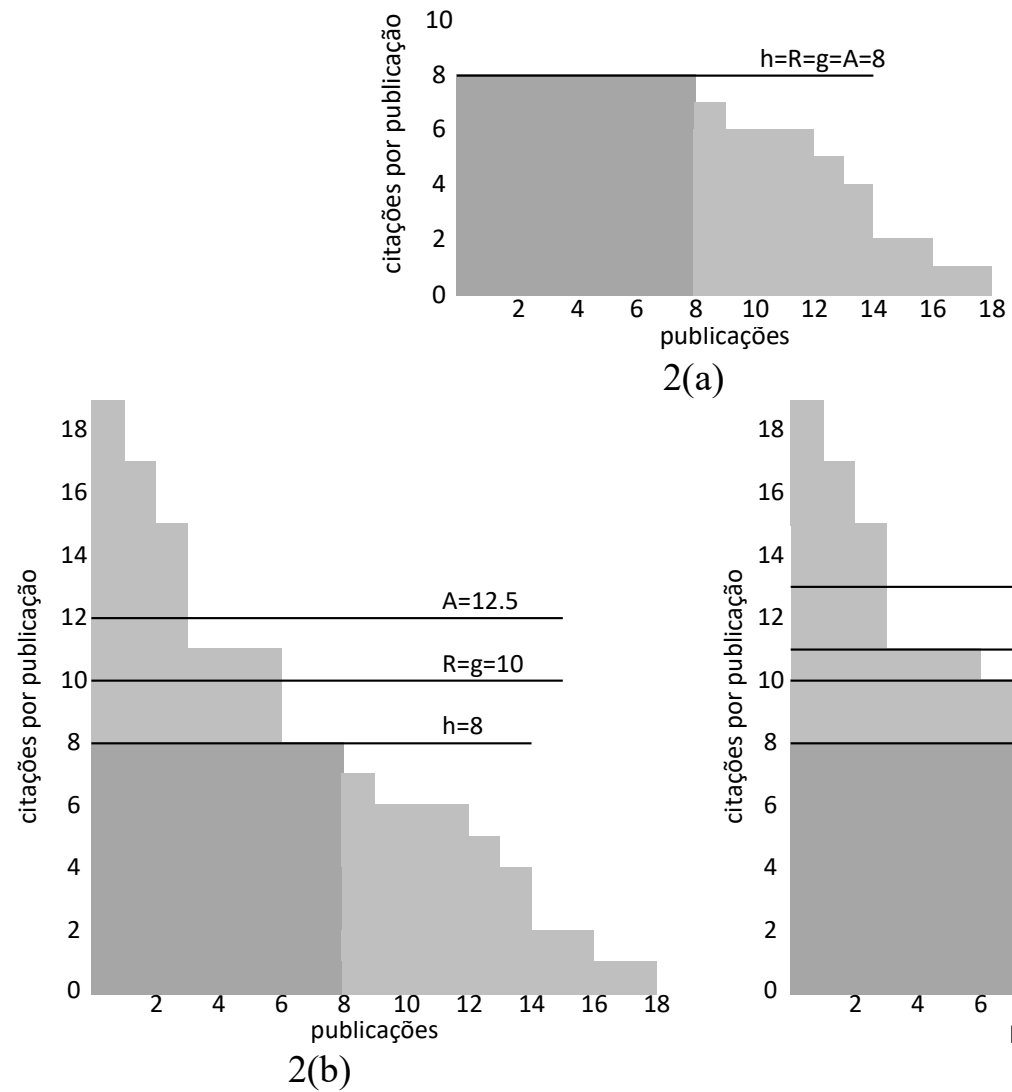

2(a)

Figura 1: Comportamento dos índices frente a um aumento do número de citações

Combinando as equações (1) e (2), observa-se que A e R são iguais somente quando $\sum_{j=1}^{h} c_{j}=h^{2}$, ou seja, quando cada publicação do núcleo tiver recebido exatamente h citações, caso em que $\mathrm{A}=\mathrm{R}=\mathrm{h}=\mathrm{g}$, conforme ilustrado em 2(a). Um aumento somente do número de citações das publicações do núcleo h eleva os valores de $\mathrm{A}$ e $\mathrm{R}$, mantendo o índice $\mathrm{h}$ constante, sendo o crescimento de A maior que o de R, como ilustrado em 2(b) e 2(c).

Já o índice g não cresce até que as 9 publicações juntas, isto é, $\mathrm{h}+1$, apresentem 81 citações. Até então, tem-se $\mathrm{A}>\mathrm{R}>\mathrm{g}$. Quando isso ocorre, no caso ilustrado, com 10 citações extras, tem-se $g=i 9 ; R=\sqrt{74}$ i 8.6; e $A=\frac{74}{8}=i$ 9.25, ou seja, A>g>R. Com novos incrementos de citações, $\mathrm{R}$ pode ser maior ou menor do que g, dependendo do número de citações acrescentadas. Entretanto, a partir da situação ilustrada em 2(c), g permanecerá maior ou igual a $\mathrm{R}$, mesmo com novas citações.

\subsection{Crescimento de Citações e Publicações - Estudo de Caso}

Para verificar o crescimento de citações e artigos em um caso real, foi realizado um estudo de caso na Escola de Engenharia da UFF. O objetivo é avaliar a produção acadêmica dos docentes do curso de pós-graduação em Engenharia de Produção desta universidade.

Para esse estudo, foi utilizado como base inicial o estudo realizado no período de 19 a 31 de outubro de 2016, no artigo De Castro Reis et al. (2017), em que foram identificados 
os docentes que atuavam no programa, através do site oficial do departamento. Em seguida, as informações com relação ao índice h e tempo de vida acadêmica de cada docente foram obtidas na base Scopus. Esse mesmo procedimento foi realizado para o presente estudo de caso, em 10 de maio de 2019, aproximadamente dois anos e meio depois.

Entende-se que os docentes que não estão listados na base Scopus não tiveram qualquer estudo publicado em veículo relevante para a comunidade científica, ou seus trabalhos não receberam citações até o momento da busca. Em ambos os casos, esses docentes foram desconsiderados do estudo.

A Tabela 1 abaixo apresenta a quantidade de artigos e citações de cada professor do programa representado no estudo. Cabe ressaltar que, com o intuito de evitar possíveis desconfortos e julgamentos precipitados, optou-se por não divulgar o nome dos docentes considerados no estudo. 


\begin{tabular}{|c|c|c|c|c|c|c|c|c|c|c|c|c|}
\hline \multirow{2}{*}{$\begin{array}{l}\text { Ordem da } \\
\text { oublicação }\end{array}$} & \multicolumn{2}{|c|}{ PRO01 } & \multicolumn{2}{|c|}{ PRO02 } & \multicolumn{2}{|c|}{ PRO03 } & \multicolumn{2}{|c|}{ PRO04 } & \multicolumn{2}{|c|}{ PRO05 } & \multicolumn{2}{|c|}{ PRO06 } \\
\hline & 2016 & 2019 & 2016 & 2019 & 2016 & 2019 & 2016 & 2019 & 2016 & 2019 & 2016 & 2019 \\
\hline 1 & 103 & 144 & 147 & 212 & 24 & 42 & 10 & 42 & 104 & 144 & 16 & 43 \\
\hline 2 & 33 & 40 & 75 & 95 & 21 & 37 & 8 & 27 & 38 & 47 & 8 & 22 \\
\hline 3 & 27 & 34 & 51 & 90 & 20 & 36 & 8 & 25 & 33 & 40 & 8 & 17 \\
\hline 4 & 25 & 30 & 44 & 86 & 18 & 29 & 7 & 17 & 27 & 34 & 5 & 14 \\
\hline 5 & 21 & 27 & 29 & 53 & 11 & 26 & 4 & 15 & 21 & 25 & 4 & 12 \\
\hline 6 & 17 & 25 & 24 & 42 & 10 & 26 & 3 & 12 & 17 & 24 & 3 & 11 \\
\hline 7 & 17 & 24 & 21 & 37 & 9 & 23 & 2 & 11 & 17 & 21 & 2 & 10 \\
\hline 8 & 16 & 21 & 20 & 36 & 9 & 22 & 2 & 10 & 12 & 17 & 2 & 10 \\
\hline 9 & 12 & 17 & 18 & 31 & 9 & 21 & 2 & 8 & 11 & 17 & 2 & 8 \\
\hline 10 & 11 & 17 & 17 & 29 & 7 & 21 & 2 & 8 & 10 & 16 & 2 & 8 \\
\hline 11 & 10 & 16 & 15 & 26 & 6 & 20 & 1 & 7 & 9 & 14 & 2 & 7 \\
\hline 12 & 9 & 14 & 14 & 26 & 6 & 19 & 1 & 7 & 9 & 14 & 1 & 6 \\
\hline 13 & 9 & 14 & 14 & 24 & 5 & 14 & 1 & 6 & 9 & 14 & 1 & 6 \\
\hline 14 & 9 & 14 & 13 & 23 & 4 & 13 & & 6 & 8 & 12 & 1 & 6 \\
\hline 15 & 8 & 14 & 13 & 23 & 4 & 13 & & 6 & 8 & 12 & 1 & 5 \\
\hline 16 & 8 & 12 & 12 & 22 & 4 & 12 & & 6 & 7 & 11 & 1 & 4 \\
\hline 17 & 7 & 11 & 12 & 21 & 3 & 12 & & 5 & 7 & 11 & 1 & 4 \\
\hline 18 & 7 & 11 & 11 & 21 & 3 & 11 & & 5 & 6 & 11 & 1 & 4 \\
\hline 19 & 7 & 11 & 11 & 19 & 3 & 11 & & 4 & 6 & 11 & 1 & 4 \\
\hline 20 & 7 & 11 & 11 & 19 & 2 & 10 & & 4 & 5 & 10 & 1 & 4 \\
\hline 21 & 6 & 10 & 10 & 18 & 2 & 10 & & 4 & 5 & 8 & 1 & 3 \\
\hline 22 & 6 & 9 & 10 & 16 & 1 & 10 & & 4 & 4 & 8 & & 3 \\
\hline 23 & 6 & 9 & 10 & 16 & 1 & 8 & & 4 & 4 & 8 & & 3 \\
\hline 24 & 6 & 8 & 9 & 16 & 1 & 8 & & 4 & 4 & 8 & & 3 \\
\hline 25 & 6 & 8 & 9 & 15 & & 7 & & 3 & 4 & 7 & & 2 \\
\hline 26 & 5 & 8 & 7 & 14 & & 7 & & 3 & 3 & 6 & & 2 \\
\hline 27 & 5 & 8 & 7 & 14 & & 6 & & 3 & 3 & 6 & & 2 \\
\hline 28 & 5 & 8 & 6 & 14 & & 6 & & 3 & 2 & 6 & & 2 \\
\hline 29 & 4 & 7 & 6 & 13 & & 5 & & 3 & 2 & 5 & & 2 \\
\hline 30 & 4 & 7 & 4 & 12 & & 5 & & 3 & 1 & 5 & & 2 \\
\hline 31 & 4 & 7 & 4 & 12 & & 5 & & 3 & 1 & 5 & & 1 \\
\hline 32 & 4 & 7 & 4 & 12 & & 5 & & 2 & 1 & 5 & & 1 \\
\hline 33 & 4 & 7 & 4 & 11 & & 5 & & 2 & & 4 & & 1 \\
\hline 34 & 4 & 6 & 4 & 11 & & 4 & & 2 & & 4 & & 1 \\
\hline 35 & 3 & 6 & 4 & 11 & & 4 & & 2 & & 4 & & 1 \\
\hline 36 & 3 & 6 & 4 & 10 & & 4 & & 2 & & 3 & & 1 \\
\hline 37 & 3 & 6 & 3 & 9 & & 4 & & 2 & & 3 & & 1 \\
\hline 38 & 2 & 5 & 2 & 8 & & 4 & & 2 & & 2 & & 1 \\
\hline 39 & 2 & 5 & 2 & 8 & & 3 & & 1 & & 2 & & 1 \\
\hline 40 & 2 & 5 & 2 & 8 & & 3 & & 1 & & 2 & & 1 \\
\hline 41 & 2 & 5 & 2 & 6 & & 2 & & 1 & & 2 & & \\
\hline 42 & 2 & 5 & 2 & 5 & & 2 & & 1 & & 2 & & \\
\hline 43 & 2 & 5 & 1 & 5 & & 2 & & 1 & & 2 & & \\
\hline 44 & 2 & 4 & 1 & 5 & & 2 & & 1 & & 2 & & \\
\hline 45 & 2 & 4 & 1 & 5 & & 1 & & 1 & & 2 & & \\
\hline 46 & 2 & 4 & 1 & 4 & & 1 & & 1 & & 1 & & \\
\hline 47 & 1 & 4 & 1 & 4 & & 1 & & 1 & & & & \\
\hline 48 & 1 & 4 & & 4 & & 1 & & 1 & & & & \\
\hline 49 & 1 & 4 & & 3 & & 1 & & 1 & & & & \\
\hline 50 & 1 & 3 & & 2 & & 1 & & 1 & & & & \\
\hline 51 & 1 & 3 & & 2 & & 1 & & & & & & \\
\hline 52 & 1 & 3 & & 2 & & 1 & & & & & & \\
\hline 53 & 1 & 3 & & 2 & & & & & & & & \\
\hline 54 & 1 & 3 & & 1 & & & & & & & & \\
\hline 55 & & 3 & & 1 & & & & & & & & \\
\hline 56 & & 3 & & 1 & & & & & & & & \\
\hline 57 & & 2 & & 1 & & & & & & & & \\
\hline 58 & & 2 & & 1 & & & & & & & & \\
\hline 59 até 67 & & 2 & & & & & & & & & & \\
\hline 68 até 77 & & 1 & & & & & & & & & & \\
\hline $\begin{array}{l}\text { TOTAL de } \\
\text { publicações }\end{array}$ & 54 & 77 & 47 & 58 & 24 & 52 & 13 & 50 & 32 & 46 & 21 & 40 \\
\hline $\begin{array}{l}\text { TOTAL de } \\
\text { citações }\end{array}$ & 467 & 716 & 692 & 1237 & 183 & 547 & 51 & 294 & 398 & 617 & 64 & 239 \\
\hline
\end{tabular}




\begin{tabular}{|c|c|c|c|c|c|c|c|c|c|c|c|c|c|c|c|c|c|c|c|c|c|c|}
\hline \multirow{2}{*}{$\begin{array}{l}\text { Ordem da } \\
\text { oublicação }\end{array}$} & \multicolumn{2}{|c|}{ PRO07 } & \multicolumn{2}{|c|}{ PRO08 } & \multicolumn{2}{|c|}{ PRO09 } & \multicolumn{2}{|c|}{ PR010 } & \multicolumn{2}{|c|}{ PR011 } & \multicolumn{2}{|c|}{ PR012 } & \multicolumn{2}{|c|}{ PR013 } & \multicolumn{2}{|c|}{ PR014 } & \multicolumn{2}{|c|}{ PR015 } & \multicolumn{2}{|c|}{ PR016 } & \multicolumn{2}{|c|}{ PR017 } \\
\hline & \begin{tabular}{|l|}
2016 \\
\end{tabular} & 2019 & 2016 & 2019 & 2016 & 2019 & 2016 & 2019 & 2016 & 2019 & 2016 & 2019 & 2016 & 2019 & \begin{tabular}{|l|}
2016 \\
\end{tabular} & \begin{tabular}{|l|}
2019 \\
\end{tabular} & 2016 & \begin{tabular}{|l|}
2019 \\
\end{tabular} & 2016 & \begin{tabular}{|l|}
2019 \\
\end{tabular} & 2016 & 2019 \\
\hline 1 & 37 & 53 & 21 & 27 & 14 & 28 & 75 & 99 & 5 & 14 & 6 & 7 & 10 & 20 & 24 & 31 & 1 & 3 & 6 & 9 & 5 & 8 \\
\hline 2 & 5 & 13 & 12 & 12 & 4 & 6 & 8 & 16 & 1 & 8 & 2 & 2 & & 7 & 1 & 12 & 1 & 2 & 1 & 3 & & \\
\hline 3 & 4 & 10 & 10 & 11 & 2 & 4 & 6 & 9 & 1 & 4 & 2 & 2 & & 7 & & 4 & 1 & 1 & & 2 & & \\
\hline 4 & 4 & 7 & 9 & 11 & 1 & 2 & 4 & 7 & 1 & 4 & 1 & 1 & & 2 & & 3 & 0 & 1 & & 1 & & \\
\hline 5 & 3 & 6 & 2 & 5 & & 2 & 3 & 5 & 1 & 4 & & 1 & & 1 & & & & & & & & \\
\hline 6 & 2 & 6 & 2 & 5 & & 2 & 3 & 5 & & 3 & & & & & & & & & & & & \\
\hline 7 & 1 & 6 & 2 & 5 & & 2 & 2 & 4 & & 2 & & & & & & & & & & & & \\
\hline 8 & 1 & 6 & 2 & 4 & & 1 & 1 & 4 & & 2 & & & & & & & & & & & & \\
\hline 9 & 1 & 4 & 1 & 3 & & 1 & 1 & 3 & & 2 & & & & & & & & & & & & \\
\hline 10 & 1 & 3 & 1 & 2 & & 1 & 1 & 2 & & 1 & & & & & & & & & & & & \\
\hline 11 & 1 & 3 & & 2 & & 1 & 1 & 1 & & 1 & & & & & & & & & & & & \\
\hline 12 & & 2 & & 2 & & 1 & 1 & 1 & & & & & & & & & & & & & & \\
\hline 13 & & 2 & & 2 & & 1 & 1 & 1 & & & & & & & & & & & & & & \\
\hline 14 & & 1 & & 1 & & 1 & & 1 & & & & & & & & & & & & & & \\
\hline 15 & & 1 & & 1 & & & & & & & & & & & & & & & & & & \\
\hline 16 & & 1 & & & & & & & & & & & & & & & & & & & & \\
\hline 17 & & 1 & & & & & & & & & & & & & & & & & & & & \\
\hline 18 & & 1 & & & & & & & & & & & & & & & & & & & & \\
\hline $\begin{array}{l}\text { TOTAL de } \\
\text { publicações }\end{array}$ & 11 & 18 & 10 & 15 & 4 & 14 & 13 & 14 & 5 & 11 & 4 & 5 & 1 & 5 & 2 & 4 & 4 & 4 & 2 & 4 & 1 & 1 \\
\hline $\begin{array}{l}\text { TOTAL de } \\
\text { citações }\end{array}$ & 60 & 126 & 62 & 93 & 21 & 53 & 107 & 158 & 9 & 45 & 11 & 13 & 10 & 37 & 25 & 50 & 3 & 7 & 7 & 15 & 5 & 8 \\
\hline
\end{tabular}

Tabela 1: Numero de publicações e suas quantidades de citações por docente avaliado.

Nesse período de 2,5 anos, observa-se que apenas 2 docentes (PRO15 e PRO17) não publicaram novos artigos que tenham sido citados, mas todos tiveram acréscimos no número de citações.

A Tabela 2, apresenta os valores dos indicadores tratados nesse estudo, com base nas informações da Tabela 1.

\begin{tabular}{l|c|c|c|c|c|c|c|c|}
\cline { 2 - 9 } & \multicolumn{2}{c|}{ Indice h } & \multicolumn{2}{c|}{ indice g } & \multicolumn{2}{c|}{ indice A } & \multicolumn{2}{c|}{ indice $\mathbf{R}$} \\
\cline { 2 - 9 } & 2016 & 2019 & 2016 & 2019 & 2016 & 2019 & 2016 & 2019 \\
\hline PRO01 & 10 & 14 & 18 & 23 & 28,2 & 31,2 & 16,8 & 20,9 \\
PRO02 & 13 & 19 & 25 & 33 & 37,6 & 48,2 & 22,1 & 30,3 \\
PRO03 & 9 & 13 & 12 & 20 & 14,6 & 25,8 & 11,4 & 18,3 \\
PRO04 & 4 & 8 & 6 & 14 & 8,3 & 19,9 & 5,7 & 12,6 \\
PRO05 & 10 & 13 & 18 & 23 & 29,0 & 32,8 & 17,0 & 20,7 \\
PRO06 & 4 & 8 & 4 & 13 & 9,3 & 17,4 & 6,1 & 11,8 \\
PRO07 & 4 & 6 & 7 & 10 & 12,5 & 15,8 & 7,1 & 9,7 \\
PRO08 & 4 & 5 & 7 & 9 & 13,0 & 13,2 & 7,2 & 8,1 \\
PRO09 & 2 & 3 & 4 & 6 & 9,0 & 12,7 & 4,2 & 6,2 \\
PRO10 & 4 & 5 & 10 & 12 & 23,3 & 27,2 & 9,6 & 11,7 \\
PRO11 & 1 & 4 & 2 & 6 & 5,0 & 7,5 & 2,2 & 5,5 \\
PRO12 & 2 & 2 & 3 & 3 & 4,0 & 4,5 & 2,8 & 3,0 \\
PRO13 & 1 & 3 & 3 & 6 & 10,0 & 11,3 & 3,2 & 5,8 \\
PRO14 & 1 & 3 & 5 & 7 & 24,0 & 15,7 & 4,9 & 6,9 \\
PRO15 & 1 & 2 & 1 & 2 & 1,0 & 2,5 & 1,0 & 2,2 \\
PRO16 & 1 & 2 & 2 & 3 & 6,0 & 6,0 & 2,4 & 3,5 \\
PRO17 & 1 & 1 & 2 & 2 & 5,0 & 8,0 & 2,2 & 2,8 \\
\hline
\end{tabular}

Tabela 2: Indicadores de cada docente

Apenas 2 dos 17 docentes mantiveram o mesmo índice h ao longo de 2,5 anos (PRO12 e PRO17). PRO17 não publicou novos artigos que tenham recebido citações, tendo apenas recebido novas citações em sua única publicação. PRO12 publicou um novo artigo e recebeu 1 nova citação, o que não foi suficiente para elevar seu índice h. Nos dois casos, os índices h e g não variaram, mas A e R sim. Isso indica que os índices A e R podem ser mais suscetíveis a variações no número de citações. Tal sensibilidade pode ser parcialmente explicada por se tratar de números reais, e não naturais, como h e g. 
Em relação às variações do índice h entre 2016 e 2019, quem, em 2016, apresentava índice $\mathrm{h}=1$, conseguiu dobrar, triplicar e até quadruplicar o valor do índice. Para os demais, o aumento do indicador variou entre $0 \%$ e $50 \%$, conforme Tabela 3 . Esse fato demonstra a natureza não linear do índice, pois quanto maior o valor do índice mais difícil é alterá-lo.

A relação demonstrada no item 2.3 desse artigo A $\geq$ g (Jin et al., 2007) pode ser confirmada na Tabela 2, sendo maior em todos os casos e igual apenas em 2016 para PRO15, que possui índice $\mathrm{h}=1$, com um artigo e uma citação, de modo que a média também é igual a 1. Pode-se observar também o ponto negativo do índice A: PRO14 publicou novos artigos e recebeu novas citações, mas o seu índice A em 2019 é menor do que em 2016, evidenciando que A não aumenta monotonicamente com o aumento de publicações e citações.

Observa-se também, no caso de PRO16, que o índice h, g e R aumentaram de 2016 para 2019, mas o indicador A não. Esse docente teve, em 2016, 1 artigo no núcleo h com 6 citações, e, em 2019, 2 artigos com 12 citações no total. Assim, o referido docente obteve $\mathrm{A}=6$ nos dois casos, mesmo tendo, em 2019, mais artigos e citações do que em 2016.

\begin{tabular}{l|cc|c|}
\cline { 2 - 3 } & \multicolumn{2}{c|}{ Indice h } & \multirow{2}{*}{ Variação } \\
\cline { 2 - 3 } & 2016 & 2019 & \\
\cline { 2 - 3 } PRO01 & 10 & 14 & $40 \%$ \\
PRO02 & 13 & 19 & $46 \%$ \\
PRO03 & 9 & 13 & $44 \%$ \\
PRO04 & 4 & 8 & $100 \%$ \\
PRO05 & 10 & 13 & $30 \%$ \\
PRO06 & 4 & 8 & $100 \%$ \\
PRO07 & 4 & 6 & $50 \%$ \\
PRO08 & 4 & 5 & $25 \%$ \\
PRO09 & 2 & 3 & $50 \%$ \\
PRO10 & 4 & 5 & $25 \%$ \\
PRO11 & 1 & 4 & $300 \%$ \\
PRO12 & 2 & 2 & $0 \%$ \\
PRO13 & 1 & 3 & $200 \%$ \\
PRO14 & 1 & 3 & $200 \%$ \\
PRO15 & 1 & 2 & $100 \%$ \\
PRO16 & 1 & 2 & $100 \%$ \\
PRO17 & 1 & 1 & $0 \%$ \\
\hline
\end{tabular}

Tabela 3: Variação do índice h

\begin{tabular}{c|c|c|c|} 
Indice h & $\begin{array}{c}\text { Ranking } \\
\mathbf{2 0 1 6}\end{array}$ & $\begin{array}{c}\text { Ranking } \\
\mathbf{2 0 1 9}\end{array}$ & $\begin{array}{c}\text { Diferença } \\
\text { dos Ranking }\end{array}$ \\
\hline PRO01 & 2 & 2 & 0 \\
PRO02 & 1 & 1 & 0 \\
PRO03 & 4 & 4 & 0 \\
PRO04 & 5 & 5 & 0 \\
PRO05 & 3 & 3 & 0 \\
PRO06 & 6 & 6 & 0 \\
PRO07 & 7 & 7 & 0 \\
PRO08 & 8 & 8 & 0 \\
PRO09 & 10 & 11 & -1 \\
PRO10 & 9 & 9 & 0 \\
PRO11 & 12 & 10 & 2 \\
PRO12 & 11 & 14 & -3 \\
PRO13 & 13 & 12 & 1 \\
PRO14 & 14 & 13 & 1 \\
PRO15 & 15 & 15 & 0 \\
PRO16 & 16 & 16 & 0 \\
PRO17 & 17 & 17 & 0 \\
& & &
\end{tabular}

Tabela 4: Ranking do índice $h$

Ao analisar o ranking apresentado na Tabela 4, verifica-se que a maioria dos docentes mantiveram a mesma posição no ranking, com apenas cinco alterações, ou seja, 70,5\% dos docentes não alteraram a posição. Isso mostra certa constância desse grupo de docentes na produção e em sua qualidade.

A mesma análise é realizada para os índices g e R, conforme Tabelas 5 e 6. 


\begin{tabular}{|c|c|c|c|c|c|c|}
\hline & \multicolumn{2}{|c|}{ Indice g } & \multirow{2}{*}{ Variação } & \multicolumn{2}{|c|}{ Tndice R } & \multirow{2}{*}{ Variação } \\
\hline & 2016 & 2019 & & 2016 & 2019 & \\
\hline PRO01 & 18 & 23 & $28 \%$ & 16,8 & 20,9 & $24 \%$ \\
\hline PRO02 & 25 & 33 & $32 \%$ & 22,1 & 30,3 & $37 \%$ \\
\hline PRO03 & 12 & 20 & $67 \%$ & 11,4 & 18,3 & $60 \%$ \\
\hline PRO04 & 6 & 14 & $133 \%$ & 5,7 & 12,6 & $120 \%$ \\
\hline PRO05 & 18 & 23 & $28 \%$ & 17,0 & 20,7 & $21 \%$ \\
\hline PRO06 & 4 & 13 & $225 \%$ & 6,1 & 11,8 & $94 \%$ \\
\hline PRO07 & 7 & 10 & $43 \%$ & 7,1 & 9,7 & $38 \%$ \\
\hline PRO08 & 7 & 9 & $29 \%$ & 7,2 & 8,1 & $13 \%$ \\
\hline PRO09 & 4 & 6 & $50 \%$ & 4,2 & 6,2 & $45 \%$ \\
\hline PRO10 & 10 & 12 & $20 \%$ & 9,6 & 11,7 & $21 \%$ \\
\hline PRO11 & 2 & 6 & $200 \%$ & 2,2 & 5,5 & $145 \%$ \\
\hline PRO12 & 3 & 3 & $0 \%$ & 2,8 & 3,0 & $6 \%$ \\
\hline PRO13 & 3 & 6 & $100 \%$ & 3,2 & 5,8 & $84 \%$ \\
\hline PRO14 & 5 & 7 & $40 \%$ & 4,9 & 6,9 & $40 \%$ \\
\hline PRO15 & 1 & 2 & $100 \%$ & 1,0 & 2,2 & $124 \%$ \\
\hline PRO16 & 2 & 3 & $50 \%$ & 2,4 & 3,5 & $41 \%$ \\
\hline PRO17 & 2 & 2 & $0 \%$ & 2,2 & 2,8 & $26 \%$ \\
\hline
\end{tabular}

\begin{tabular}{|c|c|c|c|c|c|c|}
\hline & \multicolumn{3}{|c|}{ Kankıng Indıce g } & \multicolumn{3}{|c|}{ Kankıng Indıce $\mathbf{k}$} \\
\hline & 2016 & 2019 & Diferença & 2016 & 2019 & Diferença \\
\hline PRO01 & 2 & 2 & 0 & 3 & 2 & -1 \\
\hline PRO02 & 1 & 1 & 0 & 1 & 1 & 0 \\
\hline PRO03 & 4 & 4 & 0 & 4 & 4 & 0 \\
\hline PRO04 & 8 & 5 & -3 & 9 & 5 & -4 \\
\hline PRO05 & 3 & 3 & 0 & 2 & 3 & 1 \\
\hline PRO06 & 10 & 6 & -4 & 8 & 6 & -2 \\
\hline PRO07 & 6 & 8 & 2 & 7 & 8 & 1 \\
\hline PRO08 & 7 & 9 & 2 & 6 & 9 & 3 \\
\hline PRO09 & 11 & 11 & 0 & 11 & 11 & 0 \\
\hline PRO10 & 5 & 7 & 2 & 5 & 7 & 2 \\
\hline PRO11 & 14 & 12 & -2 & 15 & 13 & -2 \\
\hline PRO12 & 12 & 14 & 2 & 13 & 15 & 2 \\
\hline PRO13 & 13 & 13 & 0 & 12 & 12 & 0 \\
\hline PRO14 & 9 & 10 & 1 & 10 & 10 & 0 \\
\hline PRO15 & 17 & 17 & 0 & 17 & 17 & 0 \\
\hline PRO16 & 15 & 15 & 0 & 14 & 14 & 0 \\
\hline PRO17 & 16 & 16 & 0 & 16 & 16 & 0 \\
\hline
\end{tabular}

Tabela 5: Variação do índice g e R

Tabela 6: Ranking do índice g e R

Em relação às variações do índice g entre 2016 e 2019, quem, em 2016, apresentava índices g entre 1 e 6 , conseguiu dobrar ou triplicar, mas nenhum conseguiu quadruplicar o valor do índice. Para os demais, o aumento do indicador variou entre $0 \%$ e $67 \%$, conforme Tabela 5. Esse fato demonstra que a dificuldade de se aumentar o valor do índice g não varia tanto quanto a do índice $h$.

Em relação ao ranking do índice g, encontram-se 8 alterações de posição, isto é, 3 alterações a mais do que as do índice h. Apenas 53\% dos docentes se mantiveram na mesma posição, nesse período.

Em relação às variações do índice $\mathrm{R}$ entre 2016 e 2019, quem, em 2016, apresentava índices $\mathrm{R}$ entre 1 e 6 , conseguiu dobrar, mas nenhum conseguiu triplicar ou quadruplicar o valor do índice. Para os demais, o aumento do indicador variou entre 6\% e 94\%. Em outras palavras, quem teve índice $\mathrm{R}$ menor não conseguiu obter uma variação tão grande quanto a de g e h, e quem tem índice $\mathrm{R}$ maior conseguiu um avanço maior do que em g e h. Esse fato demonstra que as variações de R são mais lineares do que as de g e h.

Em relação ao ranking da Tabela 6, encontram-se 9 alterações de posição, isto é, 4 alterações a mais do que as do índice h. Com relação a esse índice, apenas $47 \%$ dos docentes se mantiveram na mesma posição, nos dois anos e meio. Esse fato evidencia a maior sensibilidade do índice $\mathrm{R}$ à produção dos docentes, até mesmo quando comparada à do g. 
Não foram analisadas as variações do índice A, pois as variações nesse índice podem ser negativas, mesmo com o aumento do número de publicações e de citações, como apontado no item 2.3. Por esse motivo, o índice A somente deve ser analisado em conjunto com o índice h.

\begin{tabular}{c|c|c|c|}
\cline { 2 - 4 } & \multicolumn{3}{c|}{ VarIaçao entre kankIng } \\
\cline { 2 - 4 } & Indice h & Indice g & Indice R \\
\cline { 2 - 4 } PRO01 & $40 \%$ & $28 \%$ & $24 \%$ \\
PRO02 & $46 \%$ & $32 \%$ & $37 \%$ \\
PRO03 & $44 \%$ & $67 \%$ & $60 \%$ \\
PRO04 & $100 \%$ & $133 \%$ & $120 \%$ \\
PRO05 & $30 \%$ & $28 \%$ & $21 \%$ \\
PRO06 & $100 \%$ & $225 \%$ & $94 \%$ \\
PRO07 & $50 \%$ & $43 \%$ & $38 \%$ \\
PRO08 & $25 \%$ & $29 \%$ & $13 \%$ \\
PRO09 & $50 \%$ & $50 \%$ & $45 \%$ \\
PRO10 & $25 \%$ & $20 \%$ & $21 \%$ \\
PRO11 & $300 \%$ & $200 \%$ & $145 \%$ \\
PRO12 & $0 \%$ & $0 \%$ & $6 \%$ \\
PRO13 & $200 \%$ & $100 \%$ & $84 \%$ \\
PRO14 & $200 \%$ & $40 \%$ & $40 \%$ \\
PRO15 & $100 \%$ & $100 \%$ & $124 \%$ \\
PRO16 & $100 \%$ & $50 \%$ & $41 \%$ \\
PRO17 & $0 \%$ & $0 \%$ & $26 \%$ \\
\cline { 2 - 3 }
\end{tabular}

Tabela 7: Variação entre os Indices

\begin{tabular}{c|c|c|c|c|c|c|c|c|}
\cline { 2 - 8 } $\begin{array}{c}\text { Comparação } \\
\text { de Ranking }\end{array}$ & $\mathbf{h}$ & $\mathbf{g}$ & $\mathbf{A}$ & $\mathbf{R}$ & $\mathbf{h}$ & $\mathbf{g}$ & $\mathbf{A}$ & $\mathbf{R}$ \\
\hline PRO01 & 2 & 2 & 3 & 3 & 2 & 2 & 3 & 2 \\
PRO02 & 1 & 1 & 1 & 1 & 1 & 1 & 1 & 1 \\
PRO03 & 4 & 4 & 6 & 4 & 4 & 4 & 5 & 4 \\
PRO04 & 5 & 8 & 12 & 9 & 5 & 5 & 6 & 5 \\
PRO05 & 3 & 3 & 2 & 2 & 3 & 3 & 2 & 3 \\
PRO06 & 6 & 10 & 10 & 8 & 6 & 6 & 7 & 6 \\
PRO07 & 7 & 6 & 8 & 7 & 7 & 8 & 8 & 8 \\
PRO08 & 8 & 7 & 7 & 6 & 8 & 9 & 10 & 9 \\
PRO09 & 10 & 11 & 11 & 11 & 11 & 11 & 11 & 11 \\
PRO10 & 9 & 5 & 5 & 5 & 9 & 7 & 4 & 7 \\
PRO11 & 12 & 15 & 14 & 16 & 10 & 13 & 14 & 13 \\
PRO12 & 11 & 12 & 16 & 13 & 14 & 14 & 16 & 15 \\
PRO13 & 13 & 13 & 9 & 12 & 12 & 12 & 12 & 12 \\
PRO14 & 14 & 9 & 4 & 10 & 13 & 10 & 9 & 10 \\
PRO15 & 15 & 14 & 17 & 17 & 15 & 15 & 17 & 17 \\
PRO16 & 16 & 16 & 13 & 14 & 16 & 16 & 15 & 14 \\
PRO17 & 17 & 17 & 15 & 15 & 17 & 17 & 13 & 16 \\
\hline
\end{tabular}

Tabela 8: Ranking do índice h, g, A e R.

Ao comparar as variações de ranking para os três indicadores, conforme apresentado na Tabela 7 , observa-se que h variou entre $0 \%$ e $300 \%$, g, entre $0 \%$ e $225 \%$ e R, entre $6 \%$ e $145 \%$. Em nenhum dos casos, as três variações foram iguais. Do mesmo modo, cada indicador produz rankings diferentes, a partir dos mesmos pesquisadores e dos mesmos dados. Observa-se que apenas um docente se manteve na mesma posição em todos os casos, qual seja PRO02, que se manteve como primeiro colocado em todos os rankings. De fato, observa-se, pela Tabela 1, que tal pesquisador apresenta um número superior de citações em suas publicações, de modo geral.

Essas divergências entre os indicadores evidenciam a importância de se utilizar o método de comparação de pesquisadores mais adequado para cada situação, dependendo de suas peculiaridades. Para isso, deve-se conhecer não só os objetivos da instituição, mas também as características dos índices. Nesse sentido, é importante entender, não só como reagem frente a um aumento da produção dos pesquisadores, visto nesta seção, mas também a estabilidade dos índices, como será visto na próxima seção.

\section{ESTABILIDADE DOS ÍNDICES}

Outra característica importante para índices bibliométricos, é o seu comportamento frente a mudanças na distribuição das citações pelas publicações, sem modificar o seu total. Vale notar que tais mudanças não são possíveis na prática, no âmbito de um mesmo pesquisador, como as mudanças analisadas na seção 3. Entretanto, a distribuição de citações é considerada formalmente um critério para tais índices, conforme demonstrado por Brandão e Soares de Mello (2019) para o índice h.

De maneira geral, os índices estudados permitem certas variações na distribuição das citações dentro dos respectivos núcleos. Entretanto, o índice g permite uma variação mais significativa do que os demais índices, por dois motivos básicos. Primeiramente, o núcleo g é mais amplo do que o núcleo h, permitindo, assim, uma variação da distribuição de 
citações entre um número maior de publicações do que para os índices h, A e R. Em segundo lugar, todas as publicações do núcleo h devem ser citadas minimamente h vezes, o que não é necessário para o núcleo g. Assim, a variação da distribuição de citações deve respeitar esse limite para os índices h, A e R, mas não para o índice g.

Dessa forma, o índice g permite que as citações de seu núcleo sejam distribuídas por um número mínimo de publicações, i.e., sejam muito concentradas, desde que mantido o mesmo número de publicações citadas, sem ter o seu valor alterado. Por outro lado, quando as citações são concentradas acima do limite definido anteriormente, o núcleo h diminui, o que leva a um aumento do índice A, conforme esclarecido em 2.3. Já o comportamento do índice R não é fixo, o que será analisado adiante, pela Tabela 9.

Em contrapartida, quando as citações são dissipadas, i.e., distribuídas pelas publicações de maneira mais uniforme, o índice $\mathrm{h}$ tende a aumentar, uma vez que novas publicações podem atingir o número mínimo de citações para entrarem no núcleo, levando a uma diminuição do índice A, conforme explicado em 2.3. Nessa situação, o índice g pode diminuir, se a dissipação ultrapassar o núcleo g, de forma que um número menor de citações permaneça no núcleo. Entretanto, vale esclarecer que, mesmo com a possível redução de g e o possível aumento de $h$, frente a uma dissipação das citações, permanece válida a relação g $\geq$ h. Novamente, o índice $\mathrm{R}$ não apresenta comportamento previamente definido, como ilustrado a seguir.

A Tabela 9 ilustra o comportamento indefinido de R, frente a concentrações e dissipações das citações pelas publicações, com base em um exemplo utilizado no estudo de caso anterior, PRO11 em 2019. O núcleo h é indicado em cinza, sendo apresentados os valores dos índices h e R, bem como o total de publicações (p) e de citações (Nc), ambos mantidos fixos para todos os casos.

A segunda coluna apresenta o caso em que as citações de dentro do núcleo original sofrem uma concentração, levando a uma redução do núcleo h, bem como uma redução de $\mathrm{R}$, uma vez que o total de citações no novo núcleo é menor que o total de citações no núcleo anterior. A terceira coluna indica o caso em que as citações do núcleo são dissipadas, levando a um aumento de $h$, bem como um aumento de R, uma vez que o total de citações no novo núcleo torna-se maior do que no núcleo original.

\begin{tabular}{|c|c|c|c|c|c|}
\hline $\begin{array}{c}\text { Ordem da } \\
\text { publicação }\end{array}$ & Dados Iniciais & $\begin{array}{c}\text { Concentração } \\
\text { limitada }\end{array}$ & $\begin{array}{c}\text { Dissipação } \\
\text { limitada }\end{array}$ & $\begin{array}{c}\text { Concentração } \\
\text { geral }\end{array}$ & $\begin{array}{c}\text { Dissipação } \\
\text { Geral }\end{array}$ \\
\hline 1 & 14 & 14 & 12 & 16 & 8 \\
2 & 8 & 8 & 7 & 10 & 5 \\
3 & 4 & 5 & 6 & 6 & 5 \\
4 & 4 & 3 & 5 & 2 & 5 \\
5 & 4 & 4 & 5 & 2 & 5 \\
6 & 3 & 3 & 3 & 2 & 5 \\
7 & 2 & 2 & 2 & 2 & 3 \\
8 & 2 & 2 & 2 & 2 & 3 \\
9 & 2 & 2 & 1 & 1 & 2 \\
10 & 1 & 1 & 1 & 1 & 2 \\
11 & 1 & 1 & 1 & 1 & 2 \\
\hline p & 4 & 4 & 4 & 4 & 4 \\
Nc & 45 & 45 & 45 & 45 & 45 \\
h & 4 & 3 & 5 & 3 & 5 \\
R & 5,5 & 5,2 & 6,7 & 5,7 & 5,3 \\
\hline Resultados em comparação & $\mathrm{h}$ menor, & h maior, & h menor, & h maior, \\
com os dados originais & $\mathrm{R}$ menor & $\mathrm{R}$ maior & $\mathrm{R}$ maior & $\mathrm{R}$ menor \\
\hline
\end{tabular}

Tabela 9: Comportamento do índice $\mathrm{R}$ frente a dissipações e concentrações de citações

As demais colunas apresentam casos em que o índice R apresenta comportamentos 
contrários aos apresentados nas colunas 2 e 3. A quarta coluna apresenta uma concentração geral, em que o novo núcleo h recebe citações de publicações externas a ele, levando a uma redução de h, mas um aumento de R. Já a quinta coluna apresenta uma dissipação geral, em que o núcleo perde citações para publicações externas, levando a um aumento do índice h, mas uma redução do índice R.

Tabela 10: Base de dados e Indicadores de 4 docentes
Tabela 11: Dispersão de citações
Tabela 12: Concentração das citações 

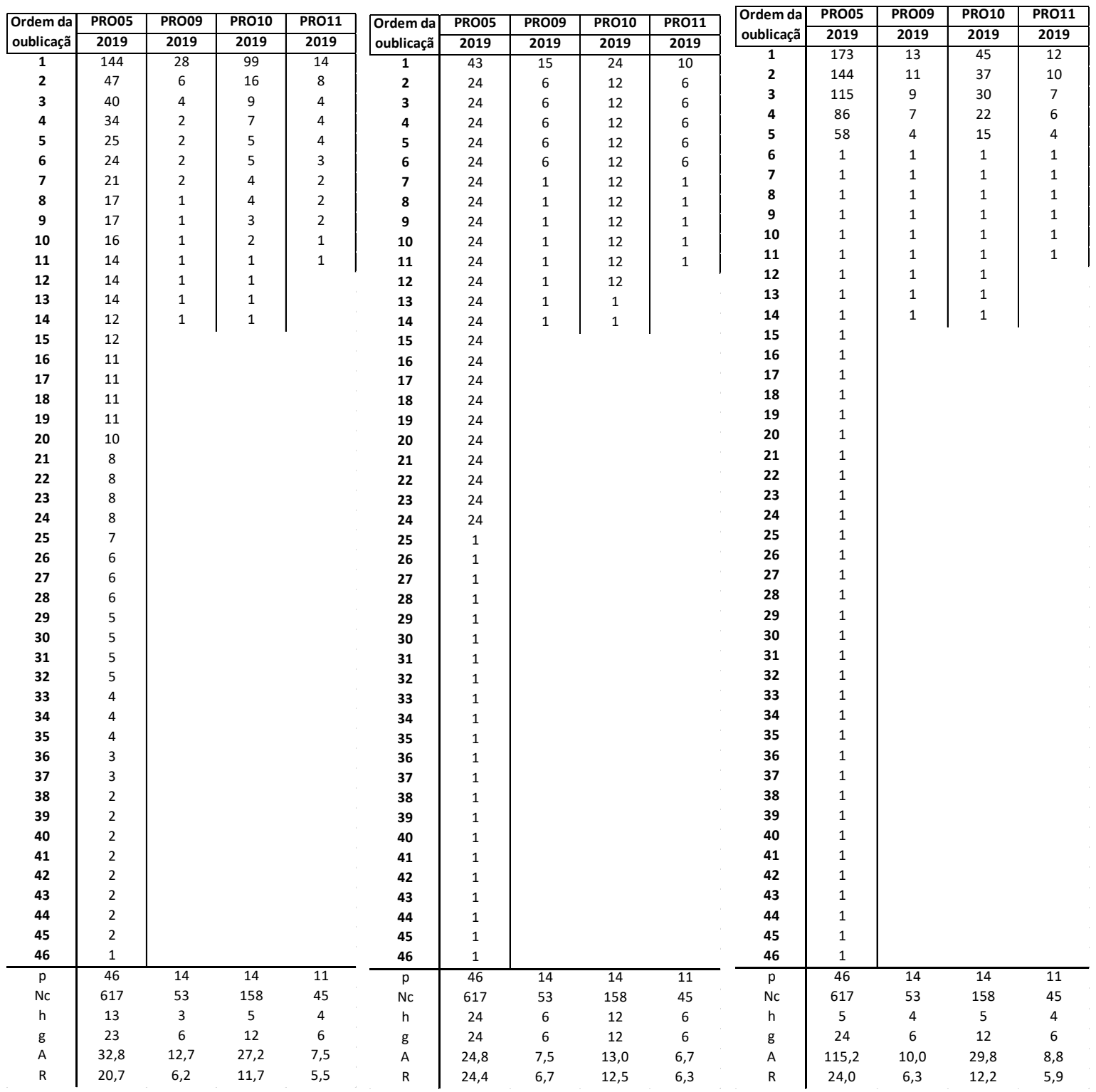

Para ilustrar a estabilidade de todos os índices analisados no presente, utilizam-se exemplos do estudo de caso da seção 3.2. A Tabela 10 considera os valores de 2019 de 4 docentes que possuem distribuições diferentes (PRO5/PRO09/PRO10/PRO11), com os respectivos números de citações para cada publicação.

As publicações são posicionadas em ordem não crescente de citações. Além do total de publicações (p) e de citações (Nc), a Tabela 10 também apresenta os índices h, g, A e $\mathrm{R}$ para cada docente.

Observa-se que a diferença entre h e g é pequena para PRO09 e PRO11 (3 e 2 respectivamente) e grande para PRO5 e PRO10 (10 e 7). Isso é consequência do fato de as citações de PRO09 e PRO11 estarem distribuídas de maneira relativamente uniforme, enquanto as citações de PRO5 e PRO10 estarem concentradas em poucas publicações. Outra consequência desse mesmo fato é a proximidade de A e h para PRO11, mas para PRO10, A é muito superior a $\mathrm{h}$.

Para analisar a estabilidade dos índices a partir dos dados da Tabela 10, as citações foram primeiramente dissipadas, i.e., distribuídas pelas publicações de maneira mais uniforme, mantendo o mesmo número de citações totais e o mesmo número de publicações citadas. Como existem diversas maneiras de se dissipar as citações, neste caso, maximiza-se o índice h. Mesmo com a definição desse objetivo, há algumas citações extras, para cada 
autor, indiferentes para o seu índice h. Essas citações foram, então, alocadas no núcleo, de modo a maximizar também, em segundo plano, os demais índices. Essa redistribuição é apresentada na Tabela 11.

Observa-se que, na Tabela $11, h=g \cong A \cong R$ para todos os pesquisadores. Na verdade, os índices não são iguais por causa das citações extras, que não influenciam o índice h, mas afetam os índices reais A e R.

Além disso, observa-se que o valor de g das Tabelas 10 e 11 é o mesmo para 3 dos 4 docentes e para PRO5 é muito próximo, o que ilustra a grande estabilidade do índice. Além disso, o índice $\mathrm{R}$ aumentou em todos os casos porque o núcleo h passou a incluir todas as citações de cada autor, exceto as citações das últimas publicações. Assim, o somatório de citações dentro do núcleo cresceu e, consequentemente, o índice $\mathrm{R}$ também. Como era esperado, o índice A reduziu para todos os pesquisadores.

Em seguida, as citações da Tabela 11 foram concentradas, i.e., redistribuídas de maneira que um menor número de publicações apresente uma maior parcela do total de citações, mas mantendo o mesmo número total de publicações citadas. Arbitrariamente, definiu-se o seguinte padrão de concentração: 30\% do total de citações de cada autor para a primeira publicação, 25\% para a segunda publicação, 20\% para a terceira, 15\% para a quarta, $10 \%$ para a quinta. Além disso, o número de publicações citadas não foi alterado, sendo, portanto, atribuída 1 citação para a oitava publicação em diante. Essa redistribuição é apresentada na Tabela 12.

Pode-se observar que o índice h ficou bastante reduzido, quando comparado às tabelas 10 e 11 . O índice A cresceu significativamente em relação às tabelas anteriores, e o índice $\mathrm{R}$ também cresceu uma vez que a soma das citações dentro do núcleo é maior, ainda que o núcleo tenha reduzido. Para $\mathrm{R}$, trata-se de situação semelhante àquela da quarta coluna da Tabela 9 de "Concentração Geral”.

Observa-se ainda que o índice g permaneceu o mesmo das Tabelas 10 e 11, o que, novamente, ilustra a grande estabilidade do índice, sendo pouco dependente da atribuição das citações. Em outras palavras, as citações recebidas por um pesquisador beneficiam, em geral, o seu índice g, não sendo muito relevante para qual publicação aquela citação está associada.

\section{CONCLUSÃO}

O presente trabalho estudou o índice h, proposto por Hirsch (2005), para medir o desempenho de pesquisadores, bem como índices tipo h, desenvolvidos na literatura para corrigir desvantagens do índice original. Foram estudados apenas índices clássicos que aumentam sensibilidade a publicações muito citadas, quais sejam, os índices g, A e R.

O presente estudo analisou o comportamento dos índices frente a aumentos do nível de produção de pesquisadores. Primeiramente, foi utilizado um exemplo numérico para analisar os efeitos de um aumento do número de citações, somente, o que ocorre na prática, principalmente após o final da carreira dos pesquisadores. Em seguida, analisou-se o comportamento desses índices em um caso real, considerando as publicações e citações dos docentes do curso Pós-Graduação de Engenharia de Produção na UFF, em dois períodos distintos. Nesse caso, o índice R mostrou-se mais sensível a variações do nível de produção dos pesquisadores. Além disso, o estudo de caso revelou que a dificuldade de se aumentar o índice R não cresce, com o aumento do índice, como o índice g ou h.

Por fim, estudou-se a estabilidade dos índices frente a variações na distribuição das citações, sem modificar o total de citações ou de publicações citadas. Verificou-se que o índice g é significativamente mais estável que todos os outros, mantendo-se constante mesmo com variações relevantes na distribuição de citações. Utilizando-se o próprio estudo 
de caso, também se verificou que, conforme esperado, os índices se aproximam quando as citações são distribuídas de maneira mais uniforme (distribuição dissipada), e se distanciam quando boa parte das citações é atribuída a um pequeno número de publicações (distribuição concentrada).

No presente trabalho, verificou-se que existem muitas diferenças no comportamento dos índices estudados. Por esse motivo, as instituições de ensino que necessitem medir a produção de pesquisadores, para fins de promoções, bolsas de estudo, pagamento de pesquisa, entre outros, devem conhecer as características dos índices disponíveis para medição, sendo este o principal objetivo do presente estudo. Trabalhos futuros podem estudar as implicações teóricas das propriedades verificadas por meio de exemplos e estudos de caso, no presente trabalho, principalmente quanto à estabilidade dos índices frente a mudanças na distribuição de citações.

\section{REFERÊNCIAS BIBLIOGRÁFICAS}

[1] Alonso, S., Cabrerizo, F.J., Herrera-Viedma, E., Herrera, F. Hg-Index: A new index to characterize the scientific output of researchers based on the h- and g-indices. Scientometrics, v. 82, n. 2, p. 391-400, 2010.

[2] Anderson, T., Hankin, R., Killworth, P. Beyond the Durfee square: Enhancing the hindex to score total publication output. Scientometrics, v. 76, n. 3, p. 577-588, 2008.

[3] Batista, P.D., Campiteli, M.G., Kinouchi, O. Is it possible to compare researchers with different scientific interests? Scientometrics, v. 68, n. 1, p. 179-189, 2006.

[4] Bornmann, L., Daniel, H.D. What do we know about the h index? Journal of the American Society for Information Science and Technology, v. 58, n. 9, p. 13811385, 2007.

[5] Brandão, L.C., Soares de Mello, J.C.C.B. Análises Comparativas entre Indicadores Bibliométricos. Pré-Anais do XLVII Simpósio Brasileiro de Pesquisa Operacional, n. 142277, ago. 2015.

[6] Brandão, L.C., Soares de Mello, J.C.C.B. A multi-criteria approach to the h-index. European Journal of Operational Research, v. 276, n. 1, p. 357-363, 2019.

[7] Braun, T., Glänzel, W., Schubert, A. A Hirsch-type index for journals. The Scientist, v. 69, n. 1, p. 169-173, 2006.

[8] BiHui, J. The AR-index: Complementing the h-index. ISSI Newsletter, v. 3, n. (1), p. 6, 2007.

[9] BiHui, J., LiMing, L., Rousseau, R., Egghe, L. The R- and AR-indices: Complementing the h-index. Chinese Science Bulletin, v. 52, n. 6, p. 855-963, 2007.

[10] Cabrerizo, F.J., Alonso, S., Herrera-Viedma, E., Herrera, F. q2-Index: Quantitative and qualitative evaluation based on the number and impact of papers in the Hirsch core. Journal of Informetrics, v. 4, n. 1, p. 23-28, 2010.

[11] De Castro Reis, J., Dos Santos Constant, R., De Mello, J.C.C.B.S. Avaliação da produção académica de docentes dos cursos de pós-graduação de engenharia da universidade federal fluminense utilizando o modelo DEA e índice h. Meta: Avaliação, v. 9, n. 27, p. 408-432, 2017. 
[12] Egghe, L. An improvement of the h-index: The g-index. ISSI Newsletter, v. 2, n. 1, p. 8-9, 2006.

[13] Egghe, L. The Hirsch index and related impact measures. Annual Review of Information Science and Technology, v. 44, p. 65-114, 2010.

[14] Egghe, L., Rousseau, R. An h-index weighted by citation impact. Information Processing and Management, v. 44, n. 2, p. 770-780, 2008.

[15] Franceschini, F., Maisano, D.A. Analysis of the Hirsch index's operational properties. European Journal of Operational Research, v. 203, n. 2, p. 494-504, 2010.

[16] Harzing, A.W., Van der Wal, R. Google Scholar as a new source for citation analysis. Ethics in Science and Environmental Politics, v. 8, n. 11, p. 61-73, 2008.

[17] Hirsch, J. E. An index to quantify an individual's scientific research output. Proceedings of the National Academy of Sciences, v. 102, n. 46, p. 16569-16572, 2005.

[18] Jin, B. H-index: An evaluation indicator proposed by scientist. Science Focus, v. 1, n. 1, p. 8-9, 2006.

[19] Jin, B., Liang, L., Rousseau, R., Egghe, L. The R- and AR-indices: Complementing the h-index. Chinese Science Bulletin, v. 52, n. 6, p. 855-863, 2007.

[20] Norris, M., Oppenheim, C. The h-index: a broad review of a new bibliometric indicator. Journal of Documentation, v. 66, n. 5, p. 681-705, 2010.

[21] Panaretos, J., Malesios, C. Assessing scientific research performance and impact with single indices. Scientometrics, v. 81, n. 3, p. 635-670, 2009.

[22] Schreiber, M. A modification of the h-index: The hm-index accounts for multiauthored manuscripts. Journal of Informetrics, v. 2, n. 3, p. 211-216, 2008.

[23] Schreiber, M. Revisiting the g-index: the average number of citations in the g-core. Journal of American Society for Information Science and Technology, v. 61, n. 1, p. 169-174, 2010a.

[24] Schreiber, M. Twenty Hirsch index variants and other indicators giving more or less preference to highly cited papers. Annalen der Physik, v. 522, n. 8, p. 536-554, 2010b.

[25] Sidiropoulos, A., Katsaros, D., Manolopoulos, Y. Hirsch h-index for disclosing latent facts in citation networks. Scientometrics, v. 72, n. 2, p. 253-280, 2007.

[26] Tol, R.S. The h-index and its alternatives: An application to the 100 most prolific economists. Scientometrics, v. 80, n. 2, p. 317-324, 2009. 